\title{
Nefropatia por IgA: análise histológica e correlação clínico- morfológica em pacientes do Estado de Minas Gerais
}

\author{
IgA nephropathy: histological analysis and clinicomorfological \\ correlation in patients from Minas Gerais State
}

\begin{abstract}
Autores
Precil Diego Miranda de Menezes Neves Juliana Reis Machado Marcos Vinícius da Silva

Débora Tavares de Resende e Silva Abate

Denise Bertulucci Rocha Rodrigues

Ana Carolina Guimarães Faleiros Marlene Antônia dos Reis

Universidade Federal do Triângulo Mineiro - UFTM.
\end{abstract}

Data de submissão: 20/07/2011 Data de aprovação: 20/03/2012

Correspondência para: Marlene Antônia dos Reis Serviço de Nefropatologia - Universidade Federal do Triângulo Mineiro Praça Manoel Terra, 330 Bairro Abadia

Uberaba - MG - Brasil CEP 38015-050

E-mail: mareis@patge. uftm.edu.br

Suporte financeiro: Fundação de Amparo à Pesquisa do Estado de Minas Gerais - FAPEMIG, Conselho Nacional de Desenvolvimento Científico eTecnológico - CNPq.

O referido estudo foi realizado no Serviço de Nefropatologia da Universidade Federal do Triângulo Mineiro - UFTM.

Os autores declaram a inexistência de conflitos de interesse.

\section{Resumo}

Introdução: A Nefropatia por IgA (NIgA) é a glomerulopatia primária mais comum. Objetivo: Classificar a NIgA segundo a nova proposta de Classificação de Oxford. Métodos: Foram analisadas biópsias do Serviço de Nefropatologia da UFTM, no período de 1996 a 2010, com diagnóstico de NIgA. Foram avaliados gênero, idade, presença de hematúria, padrões/ intensidade das lesões, deposições de IgA, IgG, IgM, Kappa, Lambda, C3, C1q e fibrinogênio. Histologicamente, as biópsias foram caracterizadas conforme a Classificação de Oxford, e realizou-se a correlação clínico-morfológica. Resultados: Das 164 biópsias avaliadas, houve predomínio do gênero masculino $(53,7 \%)$ e adulto $(93,3 \%)$. Caracterizando os pacientes conforme a classificação de Oxford, obtivemos predominância M0 $(85,3 \%)$, S1 $(53,1 \%)$, E0 $(65,2 \%)$ e T0 $(70,1 \%)$. À correção clínico-morfológica, observamos maior proteinúria $\mathrm{M} 1$ em relação a M0 ( $<<0,008)$, menor taxa de filtração glomerular estimada $(\mathrm{p}<0,001)$ e maior frequência de hipertensão $(\mathrm{p}<$ 0,001) comparando-se T0,T1 e T2. À imunofluorescência, predominância de IgA (100\% dos casos), com codeposição de C3 (99,37\% dos casos), Kappa $(96,25 \%)$, Lambda $(91,25 \%)$ e IgM $(76,92 \%)$. Foi observada correlação entre a intensidade de deposição de IgA com C3, Kappa e Lambda. Conclusão: No presente estudo, a NIgA foi predominante em homens, mais comuns foram os padrões M0, S1, E0 e T0, com maior proteinúria e aumento da hipercelularidade mesangial, além de maior prevalência de hipertensão/pior função

\section{Abstract}

Introduction: $\operatorname{Ig} \mathrm{A}$ nephropathy (IgAN) is the most common primary glomerulopathy. Objective: Classify IgAN according to the new Oxford's classification. Methods: We analyzed the renal biopsies from the Nephropathology Service of UFTM, among 1996 to 2010, with a diagnosis of IgAN. We assessed gender, age, presence of hematuria, patterns/intensity of the lesions, deposition of $\operatorname{IgA}$, IgG, IgM, Kappa, Lambda, C3, C1q and fibrinogen. Based on the histological alterations, the biopsies were characterized according to the Oxford Classification, and the clinicomorfological correlation was made. Significative results for $\mathrm{p}<0,05$. Results: A total of 164 cases biopsies, predominantly male $(53.7 \%)$ and adults $(93.3 \%)$. We characterized the patients according Oxford Classification, there was a predominance of the pattern M0 $(85,3 \%), \mathrm{S} 1(53,1 \%)$, E0 $(65,2 \%)$ e T0 $(70,1 \%)$. About the clinicomorfological correlation, we observed more severe proteinuria comparing $\mathrm{M} 1$ to $\mathrm{M} 0$ $(\mathrm{p}<0,008)$, low estimated GFR $(\mathrm{p}<$ $0,001)$ and more frequent hypertension $(\mathrm{p}<0,001)$ comparing T0, T1 e T2. On immunofluorescence, there is a predominance of IgA (100\% of cases), with codeposition of C3 (99.37\% of cases), Kappa (96.25\%), Lambda (91.25\%) and $\operatorname{IgM}(76.92 \%)$. Correlation was found between IgA intensity and C3, Kappa and Lambda. Conclusion: In this study, IgA nephropathy was predominant in males, the more frequent patterns were the M0, S1, E0 and T0, with more severe proteinuria and the enhance of mesangial hypercellularity, besides larger prevalence of hypertension/ 
renal conforme a gravidade das repercussões túbulo-intersticiais.

Palavras-chave: Glomerulonefrite por IgA. Biópsia. Patologia. worse kidney function according the tubulo-interstitial injuries.

Keywords: Glomerulonephritis, IgA. Biopsy. Pathology.

\section{INTRODUÇÃO}

A Nefropatia IgA (NIgA), ou doença de Berger, foi descrita por Berger e Hinglais em 1968 e é conhecida por ser a forma mais comum de glomerulopatia primária em todo o mundo. ${ }^{1-4}$ Além disso, possui relevante prevalência em todos os grupos étnicos, principalmente em alguns países, como Japão e Coreia, nos quais se registram as maiores incidências. Sabe-se que cerca de $50 \%$ dos casos novos de glomerulopatias e $40 \%$ de todos os pacientes com doença renal em estágio terminal são devidos à $\mathrm{NIgA}{ }^{5}$

A NIgA é caracterizada por lenta progressão clínica; em cerca de 20 anos, mais de um terço dos pacientes evoluem para a doença em estágio terminal, contribuindo substancialmente para a população de pacientes em diálise. ${ }^{1,6}$

Diante de tais estatísticas, torna-se um desafio a busca por fatores que possam predizer a evolução clínica, sendo a histopatologia uma ferramenta importante para auxiliar na estratificação clínica dos pacientes e monitorar as terapêuticas empregadas. ${ }^{7,8}$

Recentemente, a The International $\operatorname{IgA}$ Nephropathy Network juntamente à Renal Pathology Society estabeleceram e validaram uma nova classificação histopatológica para a Nefropatia por IgA, a Classificação de Oxford, ${ }^{9,10}$ que substituiu a Classificação de Haas, ${ }^{7}$ a qual vigorava desde 1997.

A partir disso, propomo-nos a avaliar a relação entre os achados histológicos e o quadro clínico do paciente. Portanto, temos cinco objetivos: 1) caracterizar clínico-epidemiologicamente os pacientes do Serviço de Nefropatologia da Universidade Federal do Triângulo Mineiro com diagnóstico de NIgA; 2) realizar a análise histopatológica das biópsias renais de tais pacientes, determinando os tipos e a intensidade das lesões de acordo com os compartimentos renais (glomérulos, túbulos, interstício e vasos) nas biópsias renais desses pacientes; 3) verificar a positividade, o local e a forma de depósito para IgA, IgG, IgM, Kappa, Lambda, C3, C1q; 4) caracterizar histologicamente as biópsias renais de acordo com a Classificação de Oxford;,9 e 5 ) realizar a correlação clínico-morfológica desses pacientes.

\section{Materiais e Métodos}

Foram utilizadas biópsias renais analisadas pelo Serviço de Nefropatologia da Universidade Federal do Triângulo Mineiro, Uberaba, Minas Gerais, no período de 1996 a 2010, de pacientes com diagnóstico compatível com Nefropatia por IgA (Doença de Berger), excluindo-se aqueles que apresentavam concomitantemente outras doenças que levam à deposição de imunoglobulinas no plano glomerular.

\section{ANÁLISE CLÍNICO-EPIDEMIOLÓGICA}

Foram registradas informações relativas aos dados epidemiológicos dos pacientes (idade e gênero), achados clínicos referentes à doença (hipertensão arterial, hematúria), histórico patológico (comorbidades prévias) e os exames laboratoriais (níveis séricos de creatinina e proteinúria de 24 horas). De posse da idade, gênero, etnia e níveis de creatinina sérica, foi calculado o Clearance de Creatinina estimado pelo método $\mathrm{MDRD},{ }^{11}$ por meio do qual os pacientes foram estratificados quanto ao grau de insuficiência renal crônica, de acordo com a taxa de filtração glomerular ( $\mathrm{mL} / \mathrm{min}$ ) em - 0: > 90 (grupos de risco para doença renal crônica, porém com ausência de lesão renal); 1: > 90 (lesão renal com função renal normal); 2: 60 - 89 (insuficiência renal leve); 3: 30 - 59 (insuficiência renal moderada); 4: 15 - 29 (insuficiência renal grave); 5: < 15 (insuficiência renal terminal ou dialítica). ${ }^{12}$

\section{ANÁLISE NA MICROSCOPIA DE LUZ}

Para a microscopia de luz, foram utilizados fragmentos de biópsia renal previamente fixados em Bouin ou paraformaldeído e emblocados em parafina. Foram confeccionadas lâminas com cortes de $2 \mu \mathrm{m}$ de espessura, e realizadas as colorações com Hematoxilina e Eosina (HE), Tricrômico de Masson (TM), Prata metenamina (PAMS) e Picro-sirius (PS). As análises das alterações foram feitas de acordo com o compartimento em que se encontra a lesão: 1) glomérulos (membrana basal, células, matriz mesangial), 2) túbulos, 3) interstício e 4) vasos, levando-se em consideração as áreas atingidas pela biópsia 
(córtex e medular) e o número de glomérulos apropriados para estudo. As lesões foram classificadas de acordo com sua intensidade em ausente $(0 \%)$, discreta $(1-25 \%)$, moderada $(26-50 \%)$ ou acentuada $(>50 \%)$, e quanto à sua extensão em focal $(<50 \%$ do parênquima) ou difusa (> $50 \%$ do parênquima).

IMUNOFLUORESCÊNCIA DIRETA PARA IGA, IgG, IgM, KaPPA, LAMBdA, C3, C10 e FIBRINOGÊNIO

Para a imunofluorescência, utilizaram-se fragmentos que foram colocados em líquido de transporte. Em seguida, os fragmentos foram congelados em nitrogênio líquido e cortados no criostato na espessura de $3 \mu \mathrm{m}$. Foram confeccionadas lâminas para cada tipo de anticorpo, anti-IgA, anti-IgG, anti-IgM, anti-Kappa, anti-Lambda, anti-C3, anti-C1q e antifibrinogênio. As secções foram incubadas com anticorpo primário conjugado com fluoresceína, em câmara escura, à temperatura ambiente, por 30 minutos. $\mathrm{O}$ excesso de anticorpo foi retirado com PBS. Posteriormente, as lâminas foram montadas com lamínula e glicerina tamponada. Essas lâminas foram visualizadas em microscópio para fluorescência e analisadas segundo a positividade, o local e a forma do depósito.

CARACTERIZAÇÃO hISTOLÓGICA DAS BIÓPSIAS RENAIS SEgundo a ClassificaÇÃo de Oxford9, 10

Após o diagnóstico de Nefropatia por IgA, as biópsias renais foram caracterizadas histologicamente de acordo com a Classificação de Oxford 2009. Nessa classificação, as variáveis observadas são a hipercelularidade mesangial, presença de glomeruloesclerose segmentar e/ou hipercelularidade endocapilar e o grau de atrofia tubular/fibrose intersticial.

A hipercelularidade mesangial é analisada da seguinte forma: $<4$ células mesangiais/área mesangial $=0 ; 4-5$ células mesangiais/área mesangial $=1 ; 6-7$ células mesangiais/área mesangial $=2 ;>8$ células mesangiais/área mesangial $=3 . \mathrm{O}$ escore de hipercelularidade mesangial é definido como a média dos escores de celularidade para todos os glomérulos. Se a média $\leq 0,5: \mathrm{M} 0$ e se a média > 0,5: M1. Além disso, se mais da metade dos glomérulos possuem mais que três células na área mesangial caracterizam-se também como M1.

Glomeruloesclerose segmentar: qualquer quantidade de tufos envolvidos por esclerose ou aderidos à parede glomerular, porém não completamente esclerosados. S0: ausente; S1: presente.

Hipercelularidade endocapilar: hipercelularidade decorrente do número aumentado de células na luz do capilar glomerular levando a estreitamento da luz. E0: ausente; E1: presente.

Atrofia tubular/fibrose intersticial: porcentagem de área cortical envolvida por atrofia tubular ou fibrose intersticial, considerando sempre a de maior intensidade. T0: $0-25 \%$; T1: $26-50 \%$; T2: > 50\%.

Após a análise das variáveis histológicas, é fornecida a Classificação final contendo os códigos (M, E, $\mathrm{S}$ e $\mathrm{T})$ apropriados.

\section{AnÁlise estatística}

A normalidade dos dados foi testada pelo teste de Kolmogorov-Smirnov. Amostras com distribuição paramétrica foram expressas em média \pm desvio-padrão e comparadas pelos testes $t$ de Student, para comparações entre dois grupos, e ANOVA para comparações entre três grupos. Dados não paramétricos foram expressos em mediana e valores de mínimo e máximo e comparados pelos testes de Mann-Withney para comparações entre dois grupos, e Kruskal-Wallis para comparação entre três grupos, com pós-teste de Dunn, Para correlação, foi utilizado teste de Spearman. As variáveis qualitativas foram expressas em porcentagens e analisadas pelo teste do Qui - quadrado ou Exato de Fischer. As diferenças foram significativas quando $\mathrm{p}<0,05$.

\section{Aspectos Éticos}

Este estudo é parte integrante do Projeto "Diagnóstico das Doenças Renais no Estado de Minas Gerais”, aprovado no Comitê de Ética da Universidade Federal do Triângulo Mineiro com o número 999.

\section{Resultados}

No acervo do Serviço de Nefropatologia da Universidade Federal do Triângulo Mineiro, havia 164 casos compatíveis com Nefropatia por IgA. Desses, $53,7 \%$ do gênero masculino e $46,3 \%$ do gênero feminino. A média de idade dos indivíduos foi de $34,93( \pm 11,9)$ anos, e a distribuição dos pacientes por faixa etária encontra-se na Figura 1. Dividindo-se por grupo etário, $6,7 \%$ eram crianças e $93,3 \%$ adultos.

Com relação aos dados clínicos e laboratoriais, proteinúria foi encontrada em $11,58 \%$ dos pacientes, sendo a mediana do valor de $1,80 \mathrm{~g} / 24 \mathrm{~h}$ $(0,3-9,4 \mathrm{~g} / 24 \mathrm{~h})$, hematúria em $62,19 \%$ e hipertensão arterial presente em $48,78 \%$. A mediana do Clearance de creatinina estimado $(\mathrm{MDRD})^{11}$ foi de $56 \mathrm{~mL} / \mathrm{kg} / 1,73 \mathrm{~m}^{2}\left(3-174 \mathrm{~mL} / \mathrm{kg} / 1,73 \mathrm{~m}^{2}\right)$. De acordo com o estágio de insuficiência renal, ${ }^{12}$ observamos 
que $26 \%$ se encontravam no Estágio I, $18,2 \%$ no Estágio II, 44,2\% no Estágio III, 3,9\% no Estágio IV e $7,7 \%$ no Estágio V.

Abordando a histopatologia, o infiltrado inflamatório foi predominantemente de células mononucleares, sendo o padrão de distribuição focal com intensidade frequentemente discreta $(32,92 \%$ dos casos). Com relação ao glomérulo, evidenciou-se o predomínio de hipercelularidade mesangial discreta (72,56\% dos casos), além da presença de esclerose segmentar com aumento de matriz mesangial, colapso de alças capilares e aderência em cápsula de Bowman em 41,46\% dos casos.

Ao classificar as biópsias renais de acordo com Oxford ${ }^{9,10}$ obtivemos as frequências de lesões

Figura 1. Distribuição dos pacientes com Nefropatia por $\lg \mathrm{A}$ de acordo com a faixa etária (em décadas).

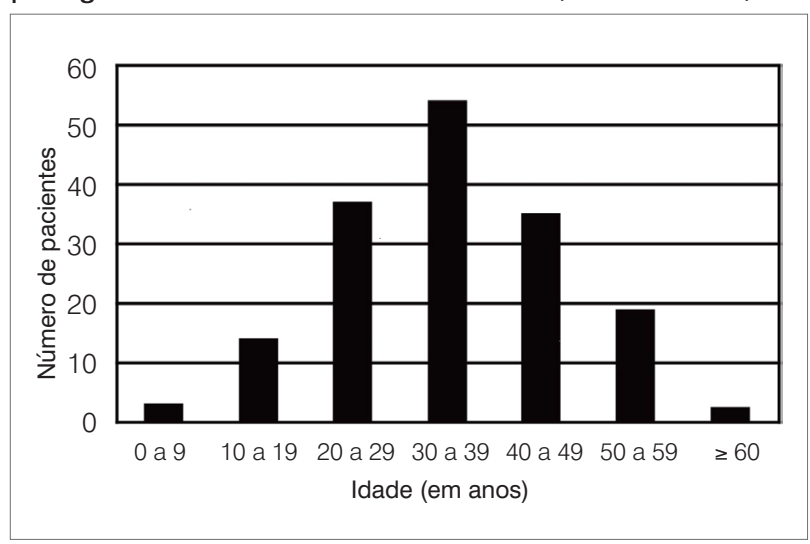

histopatológicas e classificação final, descritas na Tabela 1. A correlação clínico-morfológica pode ser observada na Tabela 2.

Em relação à imunofluorescência, a deposição predominante foi de IgA, que se deu sob intensidade acentuada em mesângio, global e difusamente, além de deposição em cilindros tubulares de mesma intensidade. Houve codeposição de IgA e C3 em 99,37\% dos casos, sendo o padrão de depósito mais comum o mesangial, acentuado, global e difuso, com depósito concomitante em parede vascular $(32,70 \%)$. Houve predominância de IgG em 22,56\% dos casos, com deposição mesangial, de discreta intensidade, global e difusa $(40,54 \%$ destes). IgM esteve presente em $76,92 \%$ dos casos,

\begin{tabular}{|c|c|c|}
\hline Tabela 1 & \multicolumn{2}{|c|}{$\begin{array}{l}\text { DISTRIBUIÇÃO DAS ALTERAÇÕES } \\
\text { HISTOPATOLÓGICAS EM BIÓPSIAS RENAIS DE } \\
\text { PACIENTES COM NIGA, DE ACORDO COM A } \\
\text { CLASSIFICAÇÃO DE OXFORD }\end{array}$} \\
\hline \multicolumn{2}{|c|}{ Hipercelularidade mesangial (M) } & $\begin{array}{l}\text { M0: } 140(85,3 \%) \\
\text { M1: } 24(14,7 \%)\end{array}$ \\
\hline \multicolumn{2}{|c|}{ Glomeruloesclerose segmentar (S) } & $\begin{array}{l}\text { S0: } 77(46,9 \%) \\
\text { S1: } 87(53,1 \%)\end{array}$ \\
\hline \multicolumn{2}{|c|}{ Hipercelularidade endocapilar (E) } & $\begin{array}{l}\text { E0: } 107(65,2 \%) \\
\text { E1: } 57(34,8 \%)\end{array}$ \\
\hline \multicolumn{2}{|c|}{$\begin{array}{l}\text { Atrofia tubular/ } \\
\text { Fibrose intersticial }(\mathrm{T})\end{array}$} & $\begin{array}{l}\text { T0: } 115(70,1 \%) \\
\text { T1: } 43(26,2 \%) \\
\text { T2: } 6(3,7 \%)\end{array}$ \\
\hline
\end{tabular}

Os dados são expressos em números e porcentagens.

Tabela 2 CorrelaÇão clínICO-MORFolóGICA dos PACIENTES COM NIGA, CONFORME A CLASSIFICAÇÃo de OXford (2009)

\begin{tabular}{|c|c|c|c|c|c|c|}
\hline Alteração histopatológica & $\begin{array}{l}\text { TFG estimada } \\
\text { pelo MDRD } \\
\text { (mL/min) }\end{array}$ & Valor $p$ & $\begin{array}{l}\text { Proteinúria de } \\
24 \mathrm{~h}(\mathrm{~g} / 24 \mathrm{~h})\end{array}$ & Valor $p$ & $\begin{array}{l}\text { Presença de } \\
\text { hipertensão } \\
\text { arterial }\end{array}$ & Valor $p$ \\
\hline \multicolumn{7}{|l|}{ Hipercelularidade mesangial } \\
\hline Mo & $56,50(3-174)$ & & $1,60(0,33-7,06)$ & & $67(45,7 \%)$ & \\
\hline M1 & $55,00(13-153)$ & 0,71 & $2,50(0,75-9,74)$ & 0,008 & $13(54,17 \%)$ & 0,66 \\
\hline \multicolumn{7}{|c|}{ Glomeruloesclerose segmentar } \\
\hline so & $54(9-174)$ & & $1,86(0,33-7,6)$ & & $35(45,5 \%)$ & \\
\hline S1 & $57,5(3-153)$ & 0,86 & $1,80(0,33-9,7)$ & 0,66 & $45(62 \%)$ & 0,44 \\
\hline \multicolumn{7}{|l|}{ Hipercelularidade endocapilar } \\
\hline E0 & $55(3-174)$ & & $1,80(0,33-9,74)$ & & $55(51,4 \%)$ & \\
\hline E1 & $60,50(11-136)$ & 0,43 & $1,80(0,4-5,0)$ & 0,94 & $25(43,8 \%)$ & 0,41 \\
\hline \multicolumn{7}{|c|}{ Atrofia tubular/ Fibrose intersticial } \\
\hline T0 & $81,77( \pm 39,53)$ & & $1,50(0,33-9,74)$ & & $43(37,4 \%)$ & \\
\hline T1 & $43,63( \pm 17,99)$ & & $2,30(0,7-6,3)$ & & $31(72,1 \%)$ & \\
\hline T2 & $25,20( \pm 13,18)$ & $<0,001$ & $2,75(0,25-7,6)$ & 0,08 & $6(100 \%)$ & $<0,001$ \\
\hline
\end{tabular}

As variáveis com distribuição normal foram expressas em média \pm desvio-padrão. As variáveis de distribuição não normal, expressas em mediana (máximo-mínimo). TFG: Taxa de Filtração Glomerular. MDRD: Modified of Diet in Renal Disease. Resultados estatisticamente significativos quando $p<0,05$. 
padrão mesangial de intensidade discreta, global e difusa ( $25 \%$ destes). C1q predominou em $15 \%$ dos casos, padrão glomerular, discreto e segmentar $(58,33 \%$ destes). Kappa esteve presente em 96,25\% dos casos, padrão mesangial acentuado, global e difuso com depósito concomitante em cilindros tubulares $(29,22 \%)$, enquanto Lambda, em 91,25\% dos casos, com padrão de deposição igual ao do Kappa, presente em 55,47\% destes. Fibrinogênio esteve presente em $8,1 \%$ dos casos. As porcentagens de codeposição à imunofluorescência estão descritas na Tabela 3. Não demonstramos variação estatisticamente significativa dos valores de creatinina sérica ou proteinúria de 24 horas de acordo com a intensidade de deposição de $\operatorname{IgA}(\mathrm{p}=0,20$ e $\mathrm{p}=0,607$, respectivamente, teste de Kruskal-Wallis).

Houve correlação entre a intensidade de deposição de IgA e de cadeia Lambda ( $p=0,0001, r=0,533$, teste de Spearman), cadeia Kappa ( $\mathrm{p}=0,0001, \mathrm{r}=0,363$, teste de Spearman), fração C3 ( $\mathrm{p}=0,0001, \mathrm{r}=0,461$, teste de Spearman), mas não com fibrinogênio, fração C1q, IgM ou IgG. Não observamos diferença estatisticamente significativa entre o número de elementos positivos à imunofluorescência e creatinina e proteinúria de 24 horas.

\section{Dıscussão}

A frequência de NIgA em biópsias renais varia amplamente ao redor do mundo, oscilando entre 2 e $52 \% .^{13}$ Tal discrepância pode ser observada quando se analisa a diferença entre as porcentagens de biópsias com NIgA de acordo com o continente. Na Ásia, por exemplo, Utsonomiya et al. ${ }^{14}$ mostraram que a NIgA corresponde a $35,4 \%$ de todas as biópsias renais no Japão. Na amostra da Austrália, Briganti et al. ${ }^{15}$ encontraram 19,3\%; Gesualdo et al. ${ }^{16}$, em amostra da Itália, 16,5\%; e Jennette et al. ${ }^{17}$, em amostra dos Estados Unidos, 6,2\%. Em nosso serviço, as biópsias de NIgA correspondem a $12,47 \%$ do acervo total e $16,15 \%$ das amostras de glomerulopatias, sendo a mais frequente das glomerulopatias primárias, seguida pela glomeruloesclerose segmentar e focal.

A predominância do sexo masculino encontrada na nossa casuística ratifica os achados da literatura, porém a proporção entre os gêneros favoreceu o sexo masculino em nossa casuística, aproximadamente $7 \%$ a mais, contrastando proporções de até $6: 1$ presentes em artigos. ${ }^{13,18}$

A NIgA pode acometer indivíduos de todas as faixas etárias, porém a maior frequência de casos é observada entre os 10 e 39 anos, com predomínio entre os 20 e 29 anos. ${ }^{19,20} \mathrm{Na}$ nossa casuística, a média de idade se

\section{Tabela 3 IMUNOFLUORESCÊNCIA: PADRÃO DE CODEPOSIÇÃO DAS BIÓPSIAS RENAIS DE PACIENTES COM NIGA}

Padrão de codeposição

Percentual

$\lg \mathrm{A}+\mathrm{C} 3 \quad 98,78$

$\lg \mathrm{A}+\lg \mathrm{M}$

78,65

$\lg \mathrm{A}+\mathrm{C} 3+\lg \mathrm{M}$

78,04

$\lg A+\lg G$

25

$\lg A+\lg G+C 3$

24,39

$\lg A+C 1 q$

14,63

$\lg \mathrm{A}+\lg \mathrm{M}+\lg \mathrm{G}+\mathrm{C} 3+\mathrm{C} 1 \mathrm{q}$ (full house)

enquadra com os achados da literatura $(34,93 \pm 11,9$ anos), porém o intervalo de idade predominante dos nossos pacientes situou-se na quarta década $(30-39$ anos) e não na terceira (20 - 29 anos).

A NIgA é caracterizada por uma apresentação clínica variável, desde hematúria assintomática, proteinúria, hipertensão, até insuficiência renal rapidamente progressiva. ${ }^{6} \mathrm{~A}$ apresentação clínica com quadro de hematúria, hipertensão e alteração dos níveis de creatinina e proteinúria apresentados pelos pacientes da nossa casuística é compatível com o que mostra a literatura, visto que tal doença pode se comportar tanto com características de síndrome nefrítica quanto de nefrótica. ${ }^{1}$ A proteinúria, habitualmente, situa-se na faixa subnefrótica, ocorrendo síndrome nefrótica em até 10,8 a $11,8 \%$ dos casos, estando tal achado da nossa casuística incluído nesse intervalo (11,58\%). A hipertensão arterial estaria presente entre 3,4 e 25,2\% dos casos, sendo esses pacientes mais prevalentes em nossa amostra (48,78\% dos pacientes) em relação à literatura. ${ }^{21,22}$ Alguns estudos identificaram que proteinúria nefrótica e hipertensão à época do diagnóstico da NIgA são fatores de mau prognóstico. ${ }^{23-26} \mathrm{No}$ presente estudo, mais de $60 \%$ dos pacientes apresentaram hematúria. Esses dados estão de acordo com alguns trabalhos cuja frequência de hematúria em pacientes com NIgA foi de 15,1 e $87,5 \% .{ }^{21,22}$

Assim como em outras glomerulopatias, por exemplo, o lúpus, a NIgA também pode cursar com vários padrões de lesões à biópsia renal, desde apenas hipercelularidade mesangial, passando pela glomerulonefrite proliferativa focal (com ou sem crescentes) até a glomerulopatia crônica esclerosante. ${ }^{7}$

No ano de 2009, os grupos de trabalho da International IgA Nephropathy Work e Renal Society of Pathology se reuniram e, de posse de dados clínicos e histopatológicos, propuseram a Classificação de Oxford, ${ }^{9,10}$ um novo sistema de classificação histológica da Nefropatia por IgA. 
Tal classificação se baseia na análise histológica da hipercelularidade mesangial, glomeruloesclerose segmentar, hipercelularidade endocapilar e atrofia tubular/fibrose intersticial, que, segundo estudos avaliados pelo grupo, seriam as alterações histológicas com maior repercussão, em longo prazo, na função renal do paciente. Após a divulgação da nova classificação, alguns artigos foram publicados com o intuito de validá-la nos vários serviços de Nefrologia ao redor do mundo, fato também almejado por nós nesse estudo.

Comparando-se nossa casuística à utilizada no estudo que gerou a Classificação de Oxford, ${ }^{9,10}$ tivemos prevalência de pacientes do gênero masculino, porém em menor cifra $(53,7$ versus $72 \%)$, média de idade similar (34 versus 30 anos), maior porcentagem de hipertensos $(48,78 \%$ versus $31 \%)$, valores de proteinúria de $24 \mathrm{~h}$ similares $(1,8$ versus $1,7 \mathrm{~g} / \mathrm{dia})$ e mediana do Clearance de creatinina estimado menor (56 versus $\left.83 \mathrm{~mL} / \mathrm{min} / 1,73 \mathrm{~m}^{2}\right)$. A população pediátrica da nossa amostra foi muito menor $(6,7$ versus $22 \%)$.

Em nossa amostra, observamos maior frequência de pacientes M0, E0, S1 e T0. Em publicação da distribuição dos padrões histológicos em biópsias de suas instituições, Kang et al. ${ }^{27}$ e Alamartine et al. ${ }^{28}$ encontraram distribuição idêntica à nossa, enquanto Halling et al. ${ }^{29}$ encontraram resultados semelhantes, divergindo apenas no fato de sua casuística apresentar maior porcentagem de pacientes sem glomeruloesclerose segmentar (S0). Já Shi et al. ${ }^{30}$ encontraram padrão de predominância M1, E1, S1 e T0 em sua população de estudo. As diferenças na distribuição dos achados histológicos e características clínicas entre os estudos podem ser devidas à heterogeneidade das populações estudadas e da proporção crianças/adultos dentre as biópsias analisadas.

Em nosso estudo, observamos declínio da taxa de filtração glomerular estimada pelo MDRD e aumento da prevalência de hipertensos com o aumento do escore $\mathrm{T}$, além de, mesmo que não estatisticamente significante, aumento da proteinúria de $24 \mathrm{~h}$. Tais achados são ratificados em outros artigos ${ }^{27-30}$ que também validam a classificação de Oxford. ${ }^{9,10}$ Além disso, estudos prévios já demonstravam que a atrofia tubular/ fibrose intersticial são preditores de pior prognóstico, refletindo em longo prazo em um rim com déficit de funcionamento por lesões crônicas. ${ }^{22,31-33}$

Nossa casuística também evidenciou maiores cifras de proteinúria de $24 \mathrm{~h}$ em pacientes M1 em relação a M0. Tais achados são ratificados nos estudos de Kang et al. ${ }^{27}$ e Halling et al. ${ }^{29}$, na própria classificação de Oxford, ${ }^{9,10}$ entretanto não foram encontrados na casuística Alamartine et al. ${ }^{28}$ e Shi et al..$^{30} \mathrm{~A}$ hipercelularidade mesangial, segundo alguns estudos prévios, poderia se relacionar a pior prognóstico de função renal por evidenciar presença de lesão renal ativa, o que, quando identificado, necessitaria de um tratamento mais potente aos pacientes. ${ }^{27,34-36}$

Apesar de outros estudos $9,10,29,30$ evidenciarem pior função renal relacionada à E1 em relação a E0 e S1 em relação a S0, em nossa casuística não evidenciamos tal fato.

Em relação à imunofluorescência, outros estudos mostram que o padrão de deposição na NIgA seria predominantemente de IgA, acompanhado por coexpressão principalmente de C3 e em menor proporção de IgG e IgM. ${ }^{37}$ Rodicio et al. ${ }^{38}$ reportaram que a deposição de IgA sozinha aconteceria em $25 \%$ dos casos, com IgG em $60-83 \%$ e IgM em $22-60 \%$. Em nosso estudo, a codeposição IgA+IgM foi mais frequente que $\mathrm{IgA}+\operatorname{IgG}$. Moriyama et al. ${ }^{39}$ demonstraram que a concomitância de deposição de IgG e IgM estaria associada à esclerose glomerular e à adesão de tufos capilares, porém tal patogênese e achados histopatológicos seriam ainda incertos e não exerceriam influência na função renal a longo prazo. A porcentagem de casos com deposição de C3 confirma a literatura prévia que evidencia tal fração do complemento em mais de $90 \%$ das biópsias. ${ }^{640}$ A porcentagem de deposição de C1q se aproxima de dados dos trabalhos. ${ }^{41,42}$ Vale ressaltar que, assim como as lesões histopatológicas, o padrão de deposição à imunofluorescência também é bastante variável ao redor do mundo e que nossos resultados expressam características das biópsias de pacientes do Estado de Minas Gerais.

Quanto às doenças de diagnóstico diferencial, temos em nosso acervo as deposições de causa secundária, como por cirrose, em que há déficit do Clearance de IgA pela falência hepática, fazendo com que haja depósito de IgA nos glomérulos, ou tal deposição faz parte do quadro clínico da doença, como na púrpura de Henoch-Schönlein e no lúpus eritematoso sistêmico. ${ }^{1}$

As informações obtidas com esse trabalho colaboram para a caracterização epidemiológica, padrão histopatológico das biópsias dos pacientes com Nefropatia por IgA no Estado de Minas Gerais. Essas informações poderiam contribuir para a possível construção de um Registro Mineiro de Glomerulopatias, como já existe no Estado de São Paulo.

\section{Conclusão}

Esse trabalho permitiu-nos conhecer o padrão de parte dos pacientes com NIgA no Brasil, padrão este 
bem mais conhecido e explorado em outros países. Concluímos que em nossa amostra, proveniente do Estado de Minas Gerais, a NIgA é a nefropatia primária mais frequente e predominante no sexo masculino associada à hematúria em mais de $60 \%$ dos casos. Segundo a Classificação de Oxford, obtivemos padrões predominantes M0, E0, S1 e T0, tendo havido na correlação clínico-morfológico maior proteinúria nos pacientes com escore $\mathrm{M} 1$ em relação a $\mathrm{M} 0$, menor Clearance de creatinina e maior frequência de hipertensão, comparando-se mutuamente os pacientes com escore T0, T1 e T2. Na imunofluorescência, houve depósito predominante de IgA, mesangial acentuado global e difuso, acompanhado por C3 e IgM.

\section{RefERÊNCIAS}

1. Donadio JV, Grande JP. IgA Nephropathy. N Engl J Med 2002;347:738-48.

2. García OG, Díaz SA, Contreras MM, López DME. Nefropatía por IgA. Rev Cubana Pediatr 2000;72:194-202.

3. Mora-Orta S, Domínguez L, Corado J, et al. Características clínico patológicas y evolución de la nefropatía por IgA en uma serie hospitalaria de niños venezolanos. Invest Clin 2007;48:167-74.

4. Llanes OB, Barry HG, Moreno J, Fuentes J. Elementos de predicción pronóstica em la nefropatía primaria por inmunoglobulina A. Rev Cubana Med 2003;42:10-4.

5. Tumlin JA, Madaio MP, Hennigar R. Idiopathic IgA Nephropathy: Pathogenesis, Histopathology, and Therapeutic Options. Clin J Am Soc Nephrol 2007;2:1054-61.

6. Roufosse CA, Cook HT. Pathological predictors of prognosis in immunoglobulin A nephropathy: a review. Curr Opin Nephrol Hypertens 2009;18:212-9.

7. Haas M. Histological subclassification of $\operatorname{IgA}$ nephropathy: a clinicopathological study of 244 cases. Am J Kidney Dis 1997;29:829-42.

8. Tomino Y. Immunoglobulin A nephropathy and chronic kidney disease. Nephrology 2010;15:23-6.

9. Cattran DC, Coppo R, Cook HT, et al. The Oxford classification of $\operatorname{IgA}$ nephropathy: rationale, clinicopathological correlations, and classification. Kidney Int 2009;76:534-45.

10. Roberts IS, Cook HT, Troyanov S, et al. The Oxford classification of IgA nephropathy: pathology definitions, correlations, and reproducibility. Kidney Int 2009;76:546-56.

11. Levey AS, Bosch JP, Lewis JB, Greene T, Rogers N, Roth D. A more accurate method to estimate glomerular filtration rate from serum creatinine: a new prediction equation. Modification of Diet in Renal Disease Study Group. Ann Intern Med 1999;130:461-70.

12. Romão Jr JE. Doença renal crônica: Definição, epidemiologia e classificação. J Bras Nefrol 2004;26:1-3.

13. Khawajah AQ, Al-Maghrabi J, Kanaan HD, AlGhamdi S. IgA nephropathy: a clinicopathologic study from two centers in Saudi Arabia. Saudi J Kidney Dis Transpl 2010;21:269-75.

14. Utsunomiya $\mathrm{Y}$, Koda $\mathrm{T}$, Kado $\mathrm{T}$, et al. Incidence of pediatric IgA nephropathy. Pediatr Nephrol 2003;18:511-5.

15. Briganti EM, Dowling J, Finlay M, et al. The incidende of biopsy-proven glomerulonephritis in Australia. Nephrol Dial Transplant 2001;16:136-7.

16. Gesualdo L, DiPalma AM, Morrone LF, Strippoli GF, Schena FP. The italian experience of the national registry of renal biopsies. Kidney Int 2004;66:890-4.

17. Jennette JC, Wall SD, Wilkman AS. Low incidence of IgA nephropathy in Blacks. Kidney International 1985;28:944-50.

18. Pessegueiro P, Barata C, Correia J. Doença de Berger: proposta de algoritmo terapêutico e fatores preditivos de insuficiência renal crônica. Acta Med Port 2003;16:261-6.

19. Koyama A. Nationwide and long-term survey of primary glomerulonephritis in Japan as observed in 1,850 biopsied cases. Research Group on Progressive Chronic Renal Disease. Nephron 1999;82:205-13.

20. Sissons JGP, Woodrow DF, Curtis JR, et al. Isolated glomerulonephritis with mesangial IgA deposits. $\mathrm{Br}$ Med J 1975;3:611-4.

21. Rivera F, López-Gómez JM, Pérez-García R. Clinicopathologic correlations of renal pathology in Spain. Kidney International 2004;66:898-904.

22. Ibels LS, Györy AZ. IgA nephropathy: analysis of the natural history, important factors in the progression of renal disease, and a review of the literature. Medicine 1994;73:79-102.

23. Johnston PA, Brown JS, Braumholtz DA, Davison AM. Clinico-pathological correlations and longterm follow-up of 253 United Kingdom patients with IgA nephropathy: A report from the MRC Glomerulonephritis Registry. QJ Med 1992;84:619-27.

24. Katafuchi R, Oh Y, Hori K, et al. An important role of glomerular segmental lesions on progression of IgA nephropathy: a multivariate analysis. Clin Nephrol 1994;41:191-8.

25. Bartosik LP, Lajoie G, Sugar L, Cattran DC. Predicting progression in $\operatorname{Ig} \mathrm{A}$ nephropathy. Am J Kidney Dis 2001;38:728-35.

26. D'Amico G, Ragni A, Gandini E, Fellin G. Typical and atypical natural history of $\operatorname{IgA}$ nephropathy in adult patients. Contrib Nephrol 1993;104:6-13.

27. Kang SH, Choi SR, Park HS, et al. The Oxford classification as a predictor of prognosis in patients with $\operatorname{IgA}$ nephropathy. Nephrol Dial Transplant 2012;27:252-8.

28. Alamartine E, Sauron C, Laurent B, Sury A, Seffert A, Mariat C. The use of the Oxford Classification of IgA nephropathy to predict renal survival. Clin J Am Soc Nephrol 2011;6:2384-8.

29. Halling SE, Söderberg MP, Berg UB. Predictors of outcome in paediatric $\operatorname{IgA}$ nephropathy with regard to clinical and histopathological variables (Oxford classification). Nephrol Dial Transplant 2012;27:715-22.

30. Shi SF, Wang SX, Jiang L, LV JC, Liu LJ, Chen YQ. Pathologic predictors of renal outcome and therapeutic 
efficacy in IgA nephropathy: validation of the Oxford classification. Clin J Am Soc Nephrol 2011;6:2175-84.

31. Tsuboi N, Kawamura T, Ishii T, Utsunomiya $\mathrm{Y}$, Hosoya T. Changes in the glomerular density and size in serial renal biopsies during the progression of IgA nephropathy. Nephrol Dial Transplant 2009;24:892-9.

32. D'Amico G. Natural history of idiopathic IgA nephropathy: role of clinical and histological prognostic factors. Am J Kidney Dis 2000;36:227-37.

33. Donadio JV, Bergstralh EJ, Offord KP, Holley KE, Spencer DC. Clinical and histopathologic associations with impaired renal function in $\operatorname{IgA}$ nephropathy. Mayo Nephrology Collaborative Group. Clin Nephrol 1994;41:65-71.

34. Roufosse CA, Cook HT. Pathologic predictors of prognosis in immu-noglobulin A nephropathy: a review. Curr Opin Nephrol Hypertension 2009;18:212-9.

35. D'Amico G. Natural history of idiopathic IgA nephropathy and factors predictive of disease outcome. Semin Nephrol 2004;24:179-96.
36. Lee HS, Lee MS, Lee SM, Lee SY, Lee ES, Lee EY, et al. Histological grading of IgA nephropathy predicting renal outcome: revisiting H.S. Lee's glomerular grading system. Nephrol Dial Transplant 2005;20:342-8.

37. Hogg RJ. Idiopathic immunoglobulin A nephropathy in children and adolescents. Pediatr Nephrol 2010;25:823-9.

38. Rodicio IL. Idiopathic IgA nephropathy. Kidney Int 1984;25:717-29.

39. Moriyama T, Shimizu A, Takei T, Uchida K, Honda K, Nitta K. Characteristics of immunoglobulin A nephropathy with mesangial immunoglobulin G and immunoglobulin $\mathrm{M}$ deposition. Nephrology 2010;15:747-54

40. Okada K, Funai M, Kawakami K, Kagami S. Yano I, Kuroda Y. IgA nephropathy in Japanese children and adults: a comparative study of clinicopathological features. Am J Nephrol 1990;10:191-7.

41. Woodroffe AJ, Gormly AA, McKenzie PE, et al. Immunologic studies in IgA nephropathy. Kidney Int 1980;18:366-74. 